\title{
David Barlow: «Panikpatienten haben allgemein Schwierigkeiten, intensive Emotionen zu bewältigen»
}

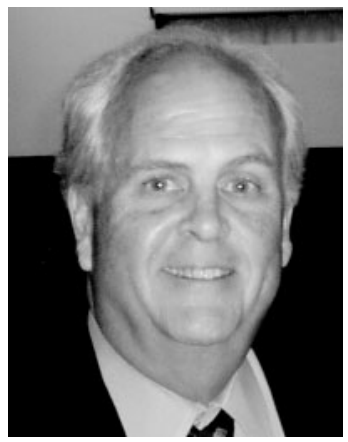

Prof. David Barlow, Boston University, hat in den USA die Entwicklung der psychologischen Behandlung von Angststörungen geprägt wie kaum ein anderer. Es ist zu einem großen Teil sein Verdienst, dass psychologische Behandlungsansätze bei Angsterkrankungen nicht nur in Psychotherapie und Psychiatrie als wesentliche Behandlungskomponente angesehen werden, sondern im gesamten Gesundheitswesen. Dazu hat er durch viele Publikationen, zum Beispiel im Journal of the American Medical Association (JAMA) beigetragen. Anlässlich einer Weiterbildungsveranstaltung in Frankfurt/Main am 24. Juni 2006 sprach Winfried Rief, Professor für Klinische Psychologie und Psychotherapie an der Universität Marburg, mit ihm über die Zukunft der Behandlung von Angsterkrankungen.

Lieber Dave, du hast ein Leben lang zu Angststörungen und deren Behandlung geforscht. Gibt es denn da überhaupt noch etwas Neues?

David Barlow: Oh ja, auf jeden Fall. Es ist zwar richtig, dass wir viel erreicht haben und viele Erkenntnisse in den letzten Jahrzehnten gewonnen haben, trotzdem sind viele Probleme noch ungelöst. Exposition zum Beispiel wirkt bei vielen $\mathrm{Pa}$ tienten, aber bei manchen Patienten und bestimmten Störungsgruppen eben auch nicht so gut, wie wir uns dies wünschen würden. Außerdem lag der Schwerpunkt der bisherigen Ansätze auf der Verhaltens- und der kognitiven Ebene; die neuen Erkenntnisse der Neurobiologie machen jedoch deutlich, dass wir auch die affektiven Komponenten bei Angsterkrankungen stärker in die Therapie integrieren müssen.

Bevor wir zur Zukunft kommen, lass uns noch kurz über die Vergangenheit sprechen. Du hast in früheren Jahren direkt mit Joseph Wolpe zusammen gearbeitet. Heute wird manchmal lächelnd auf Wolpes Ansatz der systematischen Desensibilisierung zurückgeschaut. Ist dies denn gerechtfertigt?

David Barlow: Wolpes Beitrag nicht nur zur Angstbehandlung, sondern überhaupt zur Psychotherapieforschung muss als revolutionär gewertet werden. Sicherlich sieht man heute den Ansatz der systematischen Desensibilisierung zumindest in einigen Indikationsbereichen kritischer, als Wolpe es tat. Aber Wolpe war der erste, der einen systematischen psychotherapeutischen Behandlungsleitfaden vorgeschlagen hat, der auch wissenschaftlich evaluierbar war. Er hat somit Psychotherapie überhaupt wissenschaftlich überprüfbar gemacht. Damit setzte Wolpe das Startsignal nicht nur zu modernen Ansätzen der Angstbehandlung, sondern überhaupt zur Psychotherapie als wissenschaftlich fundiertem Verfahren.

Wolpes Ansatz hat sich dann weiterentwickelt zu den Expositionsverfahren, die auch ihr sehr häufig angewandt habt. Wie kam es zu der Erkenntnis, dass auch Expositionsverfahren trotz ihrer Erfolge weiterentwickelt werden müssen?

David Barlow: Ich hatte wirklich manchmal den Eindruck, dass wir alle Spinnenphobiker in den USA in irgendeiner psychologischen Interventionsstudie zur Behandlung hatten, und auch viele Frauen mit sexuellen Ängsten konnten von den klassischen Expositionsverfahren profitieren. Aber wir haben auch festgestellt, dass der Desensibilisierungsansatz z.B. bei Agoraphobie oder bei Panikstörung nicht funktionierte. Deshalb haben wir in meiner Arbeitsgruppe den Exposi-

\begin{tabular}{llll}
\hline KARGER & @ 2006 S. Karger GmbH, Freiburg & & Prof. Dr. Winfried Rief \\
Fax +49761 4520714 & Accessible online at: & Klinische Psychologie und Psychotherapie \\
$\begin{array}{l}\text { E-mail Information@Karger.de } \\
\text { www.karger.com }\end{array}$ & www.karger.com/ver & Universität Marburg \\
& & Gutenbergstr. 18, 35032 Marburg, Deutschland \\
& & Tel. +49 6421/282-3641, Fax -8904 \\
& & E-mail rief@mailer.uni-marburg.de
\end{tabular}


tionsansatz erweitert auf die «interozeptive Exposition», die sehr viel Ähnlichkeit mit den sich parallel entwickelnden kognitiven Ansätzen der Panikbehandlung von David Clark hatte.

Der kognitiv-behaviorale Ansatz mit interozeptiver Exposition gilt als beste bekannte psychologische Therapie bei Panikstörungen. In vielen Kliniken und Praxiseinrichtungen wird deshalb der kognitiv-verhaltenstherapeutische Ansatz mit dem ebenfalls gut evaluierten psychopharmakologischen Ansatz bei Panikstörung kombiniert. Ist diese Kombination denn überhaupt sinnvoll?

David Barlow: Diese Kombination ist zwar aus klinischer Perspektive nahe liegend, wissenschaftlich gibt es jedoch keine Grundlage für eine solche Kombinationsbehandlung. Im Gegenteil, es scheint sogar, dass eine Kombinationsbehandlung einen positiven Einfluss der kognitiven Verhaltenstherapie behindern kann. Wir haben im Jahre 2000 im JAMA eine Studie mit 4 Behandlungsbedingungen veröffentlicht, die unter anderem auch eine Kombinationsbehandlung mit kognitiver Verhaltenstherapie und Imipramin enthielt. Bei der psychologischen Behandlung hatten wir deutlich unter 20\% Rückfälle im Follow-up, während wir bei der Medikamentenbehandlung über $40 \%$ Rückfälle verzeichnen mussten. Dabei gab es die meisten Rückfälle gerade bei der Kombinationsbehandlung aus Imipramin und kognitiver Verhaltenstherapie. Selbst wenn die Panikpatienten nur ein Placebo in Kombination mit kognitiver Verhaltenstherapie bekamen, schnitten sie im Langzeitverlauf besser ab als jene, die Imipramin zusätzlich bekamen. Dies spricht dafür, dass Imipramin Lernerfahrungen behindert, die für eine langfristige Stabilisierung wichtig wären.

In der Psychotherapieforschung wird unter dem Stichwort «the great debate» immer wieder die Bedeutung unspezifischer Wirkvariablen oder der Therapeut-Patient-Beziehung hervorgehoben. Manche Wissenschaftler behaupten, dass der spezifische Anteil, der auf die Therapietechnik zurückgeht, sogar weniger als $10 \%$ des Therapieerfolgs erklärt, und der Rest durch «unspezifische» Faktoren wie therapeutische Beziehung usw. zustande kommt. Ist dies auch deine Meinung?

David Barlow: Das ist meines Erachtens eine alte Fehleinschätzung, die zumindest im Bereich der Angsterkrankungen nicht mehr aufrechterhalten werden kann. Edna Foa hat bereits für die Behandlung von Zwangserkrankten beschrieben, dass $60 \%$ der Therapieerfolgsvarianz durch das systematische Vorgehen in der Behandlung erklärt würde, nur 32\% durch Patienten- und andere Variablen und $8 \%$ durch Therapeutenvariablen [Foa et al., 2005]. Bei unseren Panikstudien haben wir festgestellt, dass sich die Technik des therapeutischen Vorgehens im Prä-Post-Verlauf wirklich noch erstaunlich wenig auswirkt (10\% der Varianz); allerdings vervierfacht sich der
Einfluss der Therapietechnik, wenn man auch Follow-upDaten betrachtet. Die therapeutische Technik ist also unter Umständen weniger bedeutsam für das Urteil der Patienten bei Behandlungsende, dass ihnen die Therapie etwas gebracht habe. Ob die Therapie jedoch langfristige Erfolge hat, liegt zu einem wesentlichen Anteil an den therapeutischen Techniken und Inhalten. Die alte Aussage, dass primär die therapeutische Beziehung den Therapieerfolg prägt, ist nicht aufrechtzuerhalten.

Du hattest eingangs erwähnt, dass die neuen Erkenntnisse zur Emotionsregulation und ihre neurobiologischen Aspekte auch in der Panikbehandlung berücksichtigt werden sollten. Kannst du dies näher ausführen?

David Barlow: Wir haben festgestellt, dass Panikpatienten nicht nur negative, sondern auch positive Gefühle schlechter aushalten können. Dies spricht also für eine allgemeine Schwierigkeit, intensive Emotionen zu bewältigen. Zusätzlich erscheint uns die Erkenntnis immer wichtiger, dass viele Emotionsregulationsprobleme nicht nur im Kontext von ausgeprägten klinischen Störungen auftreten, sondern auch bei der so genannten Allgemeinbevölkerung als belastende Erfahrungen vorkommen. Etwa 10\% der Allgemeinbevölkerung gibt an, in den vergangenen 3 Monaten Panikattacken erlebt zu haben, ohne dass sie die Kriterien einer Panikstörung erfüllen. Noch häufiger sind in der Allgemeinbevölkerung depressive Einbrüche (34\%) oder intensive Phasen emotionaler Erregung (41\%). Auch wenn diese Menschen nicht die Kriterien einer Achse-1-Störung nach DSM IV erfüllen, leiden sie sehr stark unter diesen emotionalen Zuständen. Aus diesem Grund liegt uns daran, auch einen allgemeinen Ansatz zur verbesserten Emotionsregulation zu entwickeln.

Siehst du hier einen Platz für die neuen «Acceptance und Commitment»-Therapien?

David Barlow: Zu akzeptieren, dass solche intensiven emotionalen Zustände auftreten, ist sicherlich ein wichtiger Teil eines Interventionsprogramms. Hier können die Vorschläge der Akzeptanztherapie sinnvoll integriert werden. Allerdings denke ich, dass trotzdem auch die altbewährten Strategien zur Emotionsregulation eingesetzt werden müssen, um erfolgreich den Umgang mit intensiven unangenehmen Emotionen zu verbessern.

\section{Kannst du dies näher erläutern?}

David Barlow: Zur Emotionsregulation gehört auch, mehr über Gefühle, ihre natürlichen Abläufe, ihre starke erbliche Verankerung sowie Lernprozesse im Umgang mit intensiven Gefühlen zu vermitteln. Aber auch Arbeit an der antezedenten kognitiven Bewertung ist wichtig. Viele Betroffene überschätzen die Wahrscheinlichkeit von emotionalen Ausnahme- 
zuständen sowie die negativen Folgen eines intensiven emotionalen Zustandes. Deshalb sind kognitive Techniken, z.B. auch die Dekatastrophisierung wichtig. Weiterhin müssen Personen ermuntert werden, sich emotionalen Situationen zu stellen und sie nicht zu vermeiden. Zur Unterstützung können mit diesen Personen in der Therapie auch Interventionen $\mathrm{zu}$ einem verbesserten Emotionsausdruck und zur Emotionsverarbeitung durchgeführt werden.

In deinem neuen Ansatz spielt das emotionsgeleitete Verhalten (Emotion Driven Behaviors) eine große Rolle. Was ist damit gemeint?

David Barlow: Mit «Emotion Driven Behaviors» meinen wir nicht bloß Vermeidungsverhalten, sondern allgemein Verhaltenstendenzen, die durch die direkte Erfahrung einer Emotion motiviert werden. Dazu können Kontrollanrufe zu Hause zählen, wie wir sie bei der generalisierten Angststörung vorfinden, aber auch perfektionistische Verhaltensweisen oder Kontrollverhaltensweisen bei Zwangserkrankungen, das frühe Verlassen von Theatern oder Gottesdiensten bei Panikstörung oder die Hypervigilanz, wie wir sie bei allen Störungen vorfinden. So unterschiedlich diese Verhaltensweisen klingen, so ähnlich ist doch ihre Funktion im Rahmen der Emotionsregulation: sie behindern die Entwicklung von Verhaltensweisen, die mit einer besseren Bewältigung der Emotionen einhergingen. Deshalb berücksichtigen wir diese emotionsmotivierten Verhaltensweisen trotz ihrer Heterogenität bei verschiedenen emotionalen Zuständen als ein gemeinsames Merkmal in einer neu entwickelten Gruppentherapie für Personen mit emotionalen Problemen.

Was ist denn das Neue an diesem Therapieansatz?

David Barlow: Man kann uns sicher vorwerfen, dass einzelne Komponenten dieses Ansatzes nicht brandneu sind. Das ist richtig, die Stärke dieses Ansatzes ist mehr, bekannte Inter- ventionen allgemein auf den Bereich Emotionsregulation anzuwenden. Wir haben bisher für verschiedene Diagnosegruppen Behandlungsprogramme entwickelt. Die hohen Komorbiditätszahlen zeigen jedoch, dass wir unsere Behandlungsansätze nicht nur an Diagnosen festmachen können, sondern oftmals gemeinsame zugrunde liegende Prozesse beeinflussen sollten. In vielen Fällen ist die Emotionsregulation der zugrunde liegende Prozess, so dass eine therapeutische Intervention zur Verbesserung der Emotionsregulation in vielen Fällen das psychische Grundproblem trifft.

\section{Welche Bedeutung hat dies im Rahmen der allgemeinen neuro- biologischen Erkenntnisse?}

David Barlow: Bei den meisten emotionalen Störungen, unabhängig davon, ob es sich um Angst, Depression, posttraumatische Belastungsstörungen oder Zwangsstörungen handelt, sind Aktivierungsprozesse der Amygdala beteiligt. Wir wissen heute, dass nicht nur die Pharmakotherapie diese Aktivierungsprozesse beeinflussen kann, sondern auch die kognitive Verhaltenstherapie. Wir müssten deshalb versuchen, die kognitive Verhaltenstherapie so zu verbessern, dass sie Aktivierungsprozesse der Amygdala möglichst optimal zu normalisieren hilft.

Die Erforschung der Angststörungen: Immer noch kein Ende in Sicht?

David Barlow: Nein, Angststörungen sind ein faszinierender Bereich der klinischen Psychologie, in dem auch in Zukunft noch viele Fragen offen sein werden.

\section{Literatur}

Foa EB, Liebowitz MR, Kozak MJ, et al.: Randomized, placebo-controlled trial of exposure and ritual prevention, clomipramine, and their combination in the treatment of obsessive-compulsive disorder. Am J Psychiatry 2005;162(1):151-61. 